\title{
Telemedicine during COVID-19: a survey of Health Care Professionals' perceptions
}

\author{
Abdula Elawady ${ }^{1}$, Ahmed Khalil ${ }^{2,3}$, Omar Assaf ${ }^{1}$, Samirah Toure², Christopher Cassidy ${ }^{1}$ \\ ${ }^{1}$ Department of Cardiology, Lancashire Cardiac Centre, Blackpool Teaching Hospitals National Health Service \\ Foundation Trust, Blackpool, UK; ${ }^{2}$ Department of Obstetrics and Gynecology, Dartford and Gravesham National \\ Health Service Trust, Darenth Wood Road, Dartford, Kent, UK; ${ }^{3}$ Department of Obstetrics and Gynecology, Benha \\ University, Egypt
}

\begin{abstract}
The National Health Service (NHS) has rapidly adopted telemedicine solutions as an alternative to face-to-face consultations during the COVID-19 pandemic. The majority of HCPs (Healthcare Professionals) were unfamiliar with Telemedicine prior to the current pandemic. Remote consultation is expected to continue for the foreseeable future, thus we designed this survey. A survey designed to evaluate the use of telephone consultation by HCPs, assessing its implementation, challenges and drawbacks. A web link survey conducted through SurveyMonkey was sent to HCPs across six UK Trusts the period of May 2020. The survey received 114 responses (84\%) being doctors. $95 \%$ of respondents had not received training prior to engaging in telemedicine consultations. $64 \%$ were unaware of the updated General Medical Council guidance concerning
\end{abstract}

Correspondence: Abdula Elawady, Department of Cardiology, Lancashire Cardiac Centre, Blackpool Teaching Hospitals NHS Foundation Trust, Whinney Heys Rd, Blackpool FY3 8NR, UK. Tel. +44.7576933384. E-mail: abdula.elawady@nhs.net

Key words: COVID-19; survey; remote consultation; telemedicine.

Contributions: AE, AK, conceptualization, methodology, investigation, data collection, formal analysis, writing - original draft, writing review and editing, final approval; OA, ST, data collection, writing review and editing, final approval; CC, conceptualization, writing review and editing, supervision, final approval. All the authors have read and approved the final version of the manuscript and agreed to be accountable for all aspects of the work.

Conflict of interest: The authors declare no conflict of interest.

Ethics approval: not applicable

Funding: None.

Received for publication: 24 July 2020.

Accepted for publication: 4 August 2020.

${ }^{\circ}$ Copyright: the Author(s), 2020

Licensee PAGEPress, Italy

Monaldi Archives for Chest Disease 2020; 90:1528

doi: $10.4081 /$ monaldi.2020.1528

This article is distributed under the terms of the Creative Commons Attribution Noncommercial License (by-nc 4.0) which permits any noncommercial use, distribution, and reproduction in any medium, provided the original author(s) and source are credited. remote consultations. The most common barrier in remote consultation was the inability to access patient records raised by $37 \%$ of respondents. However, $73 \%$ of respondents felt that patients understood their medical condition and the instructions given to them over the phone, and $70 \%$ agreed that videoconference consultations would add to patients care. Telemedicine can be used for selected groups of patients in the post COVID-19 era, and the HCPs carrying that should have the sufficient experience and knowledge expected to operate these clinics.

\section{Introduction}

Telemedicine is described by the World Health Organisation (WHO) as: "The delivery of health care services, where distance is a critical factor, by all healthcare professionals using information and communication technologies for the exchange of valid information for diagnosis, treatment and prevention of disease and injuries, research and evaluation, and for the continuing education of health care providers, all in the interests of advancing the health of individuals and their communities" [1].

On the $11^{\text {th }}$ of March 2020, the WHO declared the novel coronavirus (COVID-19) outbreak as a global pandemic. The WHO Director General Dr. Tedros Ghebreyesus has called on all nations and said "We should double down, we should be more aggressive" [2] in order to contain the spread of the virus. In the absence of a known medical intervention the principle strategy adopted to halt the spread of the virus was social distancing measures and a complete lockdown across many nations [3].

The National Health Service (NHS) has rapidly adopted telemedicine solutions as an alternative to face-to-face consultations. This has helped Healthcare Professionals (HCPs) to continue the provision of clinical support to patients to some extent whilst adhering to National distancing requirements. The majority of HCPs were unfamiliar with Telemedicine prior to the current pandemic. As we are expecting remote consultation to continue for the foreseeable future, we designed a generic survey to understand the perceptions of HCPs and challenges encountered when using this technology.

\section{Aims}

The aim of this survey was to evaluate the use of telephone consultation in clinics by healthcare professionals, assessing its implementation, challenges and drawbacks. 


\section{Methodology}

A web link survey conducted through SurveyMonkey was sent to all HCPs across six UK Trusts in the North West and South East regions for the period of May 2020 (Supplementary Material). The questionnaire comprised of 9 questions, which had been proposed by the authors to facilitate its completion within 5 minutes. An email was sent explaining the aim of the survey with a link to the questionnaire, through medical education and rota co-ordinators in each Trust. Responses to the survey were rated using the Likert scale. The results from the free text box were categorized and coded by the authors.

\section{Results}

The survey received 114 responses, $18(16 \%)$ of respondents were specialist nurses and midwives with the remaining $96(84 \%)$ being doctors. Of the doctors that responded, 35 (31\% of all respondents) were Consultants, 25 (22\%) were Staff grade doctors, $23(20 \%)$ were trainee registrars and $13(11 \%)$ were core specialty trainees. The majority of respondents worked in medicine, surgery and obstetrics and gynaecology. Only 6\% (7 respondents) were from anaesthetics, paediatrics or community based.

Regarding training and awareness of the guidance for telemedicine, 95\% (108/114) of respondents had not received training prior to engaging in telemedicine consultations. Sixty-four percent (73/114) of respondents were unaware of the updated General Medical Council (GMC) guidance concerning remote consultations.

When asked about barriers and challenges with consultations, respondents were able to choose more than one answer. The results are displayed in Figure 1. Twenty-six (23\%) felt there were no barriers in the use of telemedicine consultations. The most common barrier was the inability to access patient records raised by 43 respondents $(37 \%)$. A common additional concern raised in the free-text responses was the inability to perform an examination or engage in non-verbal communication hindering the consultation.

The survey asked whether clinicians felt that patients under-

\section{Question: Have you come across any barriers or challenges in the assessment of patients over the phone?}

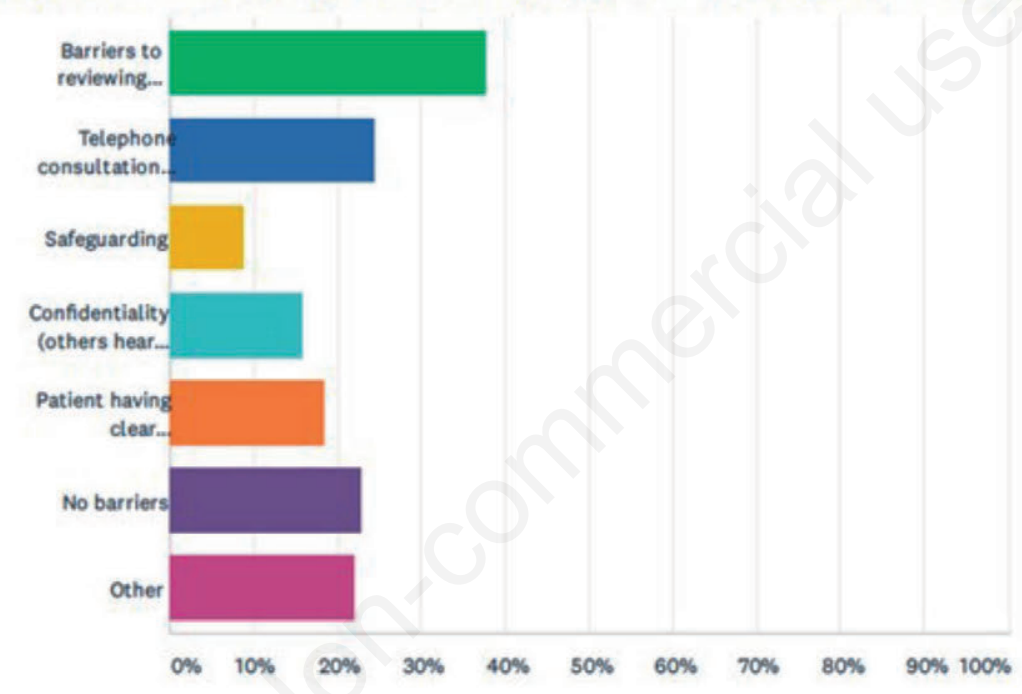

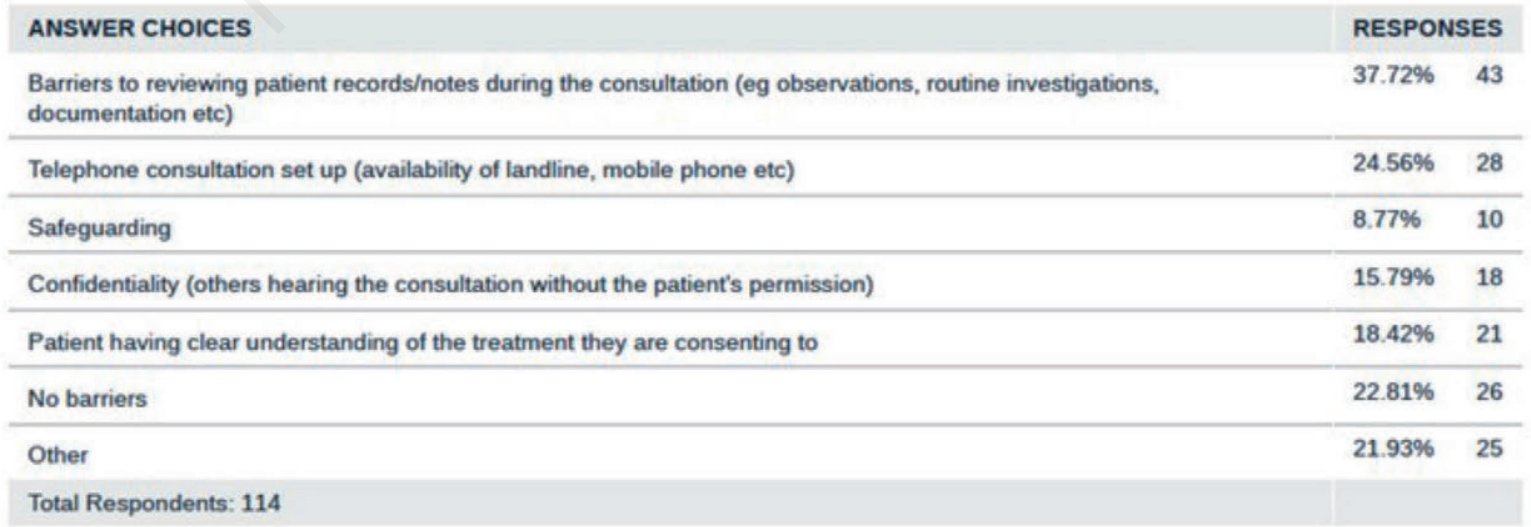

Figure 1. Barriers and challenges with consultations. 
stood their medical condition and the instructions given to them over the phone (Figure 2$) .83(73 \%)$ of respondents agreed to that statement, whilst only $12(10 \%)$ were not in agreement. Of note, 66 $(58 \%)$ of respondents thought patients were satisfied with the care provided via telephone consultation (Figure 3).

Results from the survey showed that $44 \%$ of respondents felt that less patients are discharged, with more follow up appointments were arranged when using telephone consultations. Twenty-four percent disagreed with this statement whilst $32 \%$ were impartial to the suggestion (Figure 4). It was asked whether videoconference consultations would add to patients care rather than just telephone consultations (Figure 5): 80 (70\%) respondents agreed with this statement. On asking about the preferred methods of communicating abnormal results, $68(60 \%)$ preferred face to face communication, whilst 30 (26\%) chose telephone consultation, and $6(5 \%)$ preferred to send a written report and $3(3 \%)$ secure email. The remaining $7(6 \%)$ had no preference with the choice of communication.

In assessing the impact of COVID-19 on the patient willingness to present to the $\mathrm{A} \& \mathrm{E}$ when warning signs related to their medical condition developed. Fifty-seven (51\%) of the respondents agreed with the statement that these patients were presenting late, $42(38 \%)$ had an indifferent response to this statement, whilst 3 skipped it, and $12(11 \%)$ disagreed.

\section{Discussion}

\section{Summary of findings}

To the best of our knowledge, this is the first published survey designed for health care professionals, examining the impact of telemedicine use during the COVID-19 pandemic. Our survey was based on identifying the technical and adaptive challenges across the NHS (National Health Service). Guidance released by the GMC and various Royal Colleges addresses a number of requirements when using telemedicine. These include initial identification of patients who qualify for remote consultations, confirming the identity of patients over the phone, consenting patients for telemedicine, making them aware that there are limitations of clinical assessment and assessing their capacity to make a decision about treatments made. In addition to the GMC, defence unions such as the MDU have facilitated webinars [4] to help support clinicians when delving into this realm of consultation. Surprisingly, $64 \%$ of HCPs are not aware of the updated guidance regarding remote consultations. Furthermore, only $5 \%$ have received some sort of training prior to the start of telephone consultations. The amount of guidance, policies and procedures released during the pandemic was quite overwhelming for all HCPs.

The GMC have created a flowchart [5] to aid decision-making when determining suitability for telemedicine consultation. This could be used as the basis for triage of patients to telemedicine versus face to face consultation. The lack of training and guidance can pose a medico-legal burden as well as patient safety concern, the implications of which have not yet been clearly described. At present NHS England has allowed the option to pause complaints for 3 months during the pandemic [6]. A general idea of the various barriers to communication has been presented in the results. Some of the more striking features from the survey originate from the free text responses, for example "as a geriatrician our patients often have hearing impairment or don't have access to internet for video consultation".

A large proportion of our cohort (70\%) believes that videoconferencing exceeds telephone consultations in regards to effective communication. The hypothesis around this is structured around

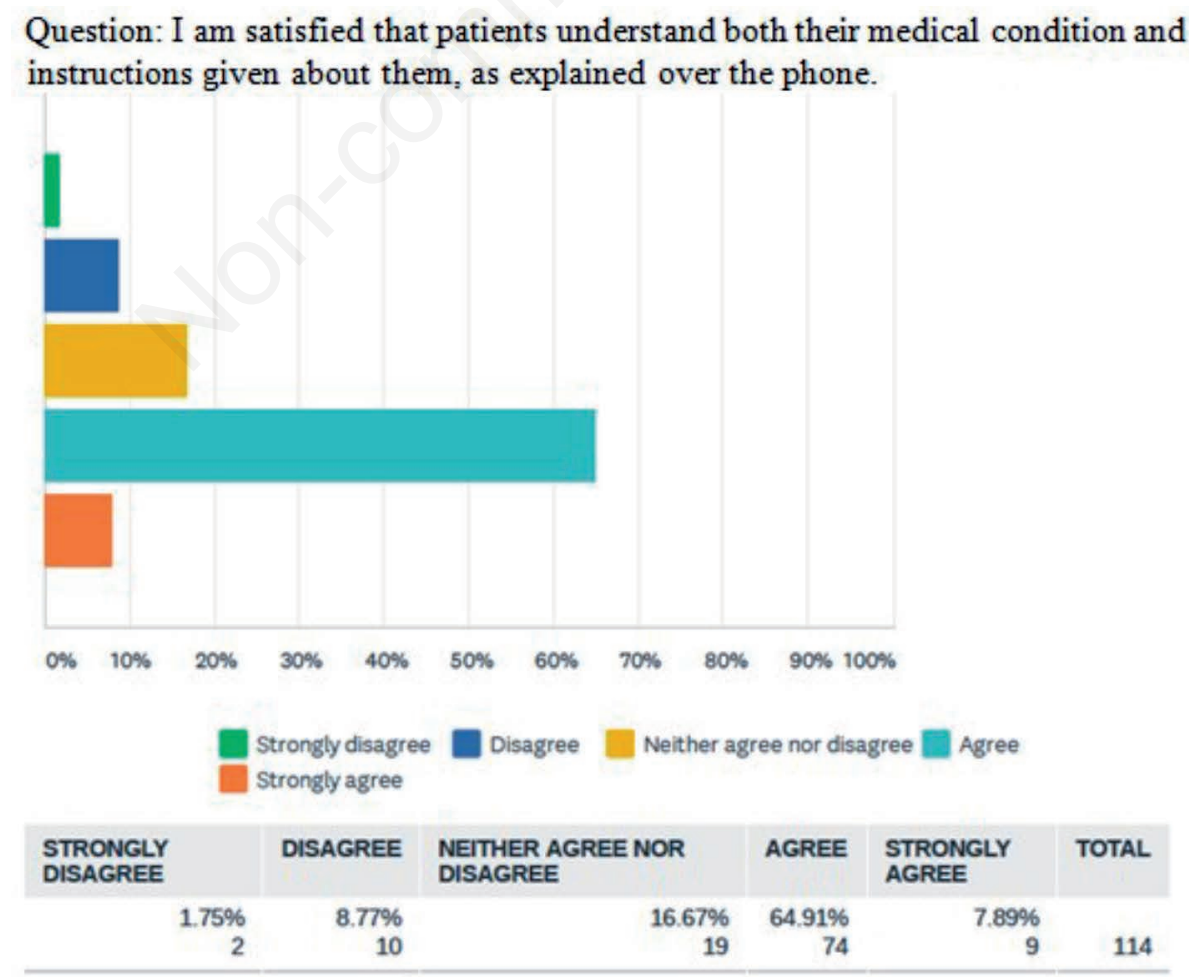

Figure 2. Patient's understanding of their medical conditions and of the instructions given. 
Question: Patients are satisfied with the level of care provided over the phone.

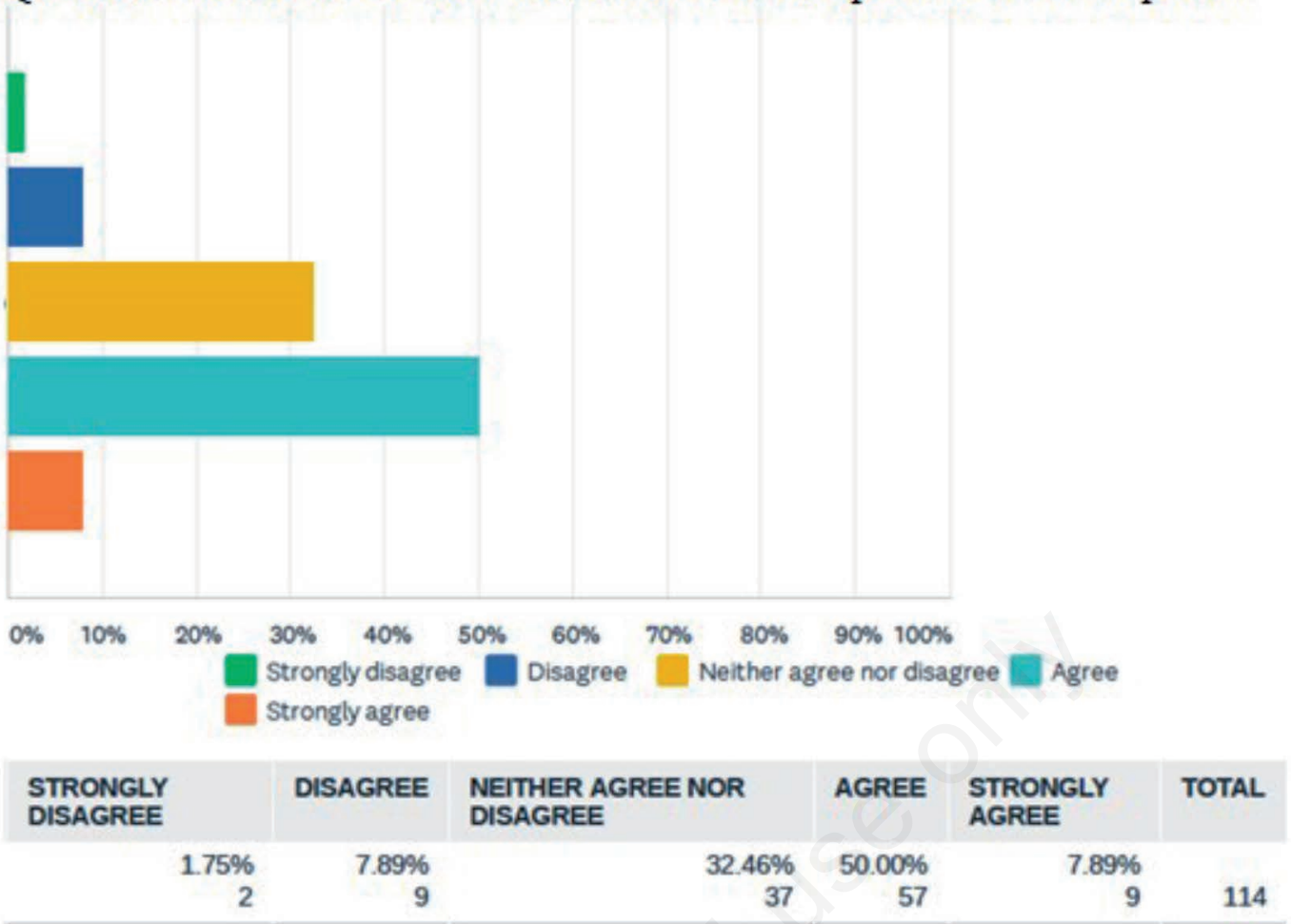

Figure 3. Patients' satisfaction with the level of care.

Question: The number of patients discharged from the telephone clinics is less, with more follow up appointments arranged.

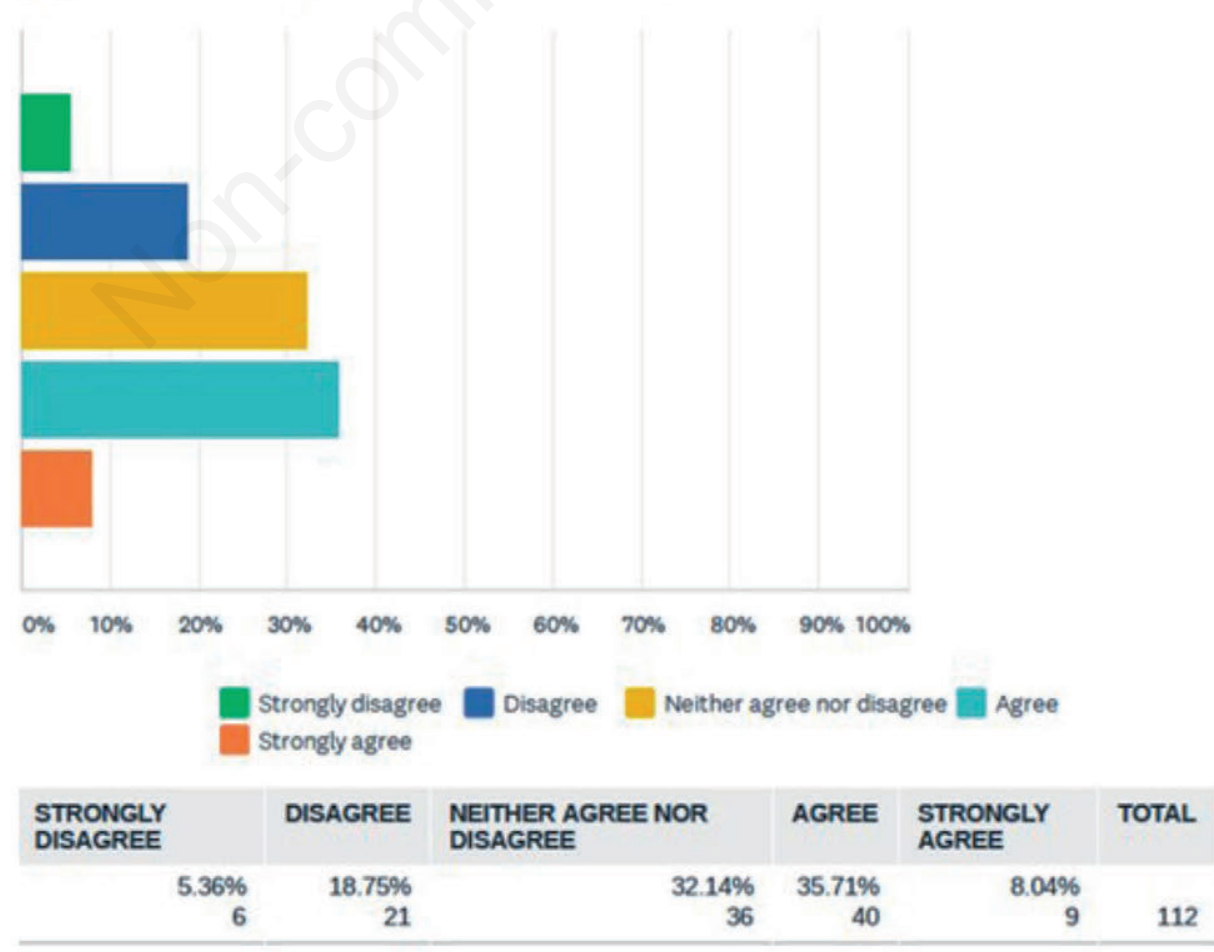

Figure 4. Discharge rates and follow up appointments. 
the availability of non-verbal communication, the possibility of limited examination, and the ability to deliver news to patients more effectively.

The challenges facing videoconferencing include but are not limited to; lack of integration with the infrastructure, patients' lack of technical skills, expensive technology for both patients and hospitals, and privacy concerns. On balance, telemedicine has improved access to services by increasing care delivery and reducing health-care costs [7]. The adaptive challenges are more difficult to identify. They are concerned with patients and HCPs adopting the idea of telemedicine. This includes learning new attitudes and behaviours within their daily practice. The results of the survey are promising, with more than $60 \%$ of HCP's being satisfied with the level of care provided. Of note General practice has more experience with telemedicine and it has shown that there is a reluctance to adopt certain telemedicine methods due to concerns around risk and workload burden [8].

Discharge rates from clinics were another indicator of confidence in telemedicine. One of the positive free text responses "If consultant led or experienced, well supported registrar, discharge rate may even be higher". On the other hand: "Some patients have been given follow up appointments they usually wouldn't have them. Typically, it has been ones listed for procedures. I have given them a further follow up to discuss any concerns, due to the amount of information given over the phone".

\section{Strengths and limitations}

The points of strengths identified in this study: i) This is the first survey to look at the use of telemedicine during the COVID-19 pandemic amongst HCP's in the UK and the challenges its use has raised; ii) The responses were collected from six different NHS trusts, with it being a cross study representing various clinical grades (consultants, staff grade doctors and trainees) and specialized nurses; iii) The service was target oriented, with resultant recommendations, if followed can improve the level of service provided.

On balance the points of weakness identified were as follows: i) The results cannot be generalised to the wider healthcare population given the small survey response number; ii) The inability to determine the response rate, as a general email was sent with the web link attached. Given the huge amount of emails sent, many clinicians may have missed the email or did not have time to fill the survey; iii) The vast majority of responses are from medicine, surgery and women health.

\section{Comparison with coexisting literature}

The use of alternatives to face to face consultations has been recognised and described in the literature. The magnitude of remote consultation activity is difficult to estimate $[9,10]$. Various studies in developed as well as in developing countries examined the acceptance of HCPs and patients to the concept of remote consultations especially in rural areas [11,12]. A Questionnaire based assessment in Spain [11] and another in Uganda [12] confirmed that the HCPs are willing to implement remote consultations as an alternative to face to face consultations. Our survey showed similar results.

Prior to the COVID-19 pandemic, Health Education England in 2016 demanded more evidence before recommending remote consultation in primary care [13]. Furthermore, a General practice (GP) based Survey (in 2018) concluded that online consultations via E-mail was effective and convenient to certain patient groups with non-urgent problems, which is similarly reflected in our survey that certain specialities might have difficulties at first going through with this novel approach [14].

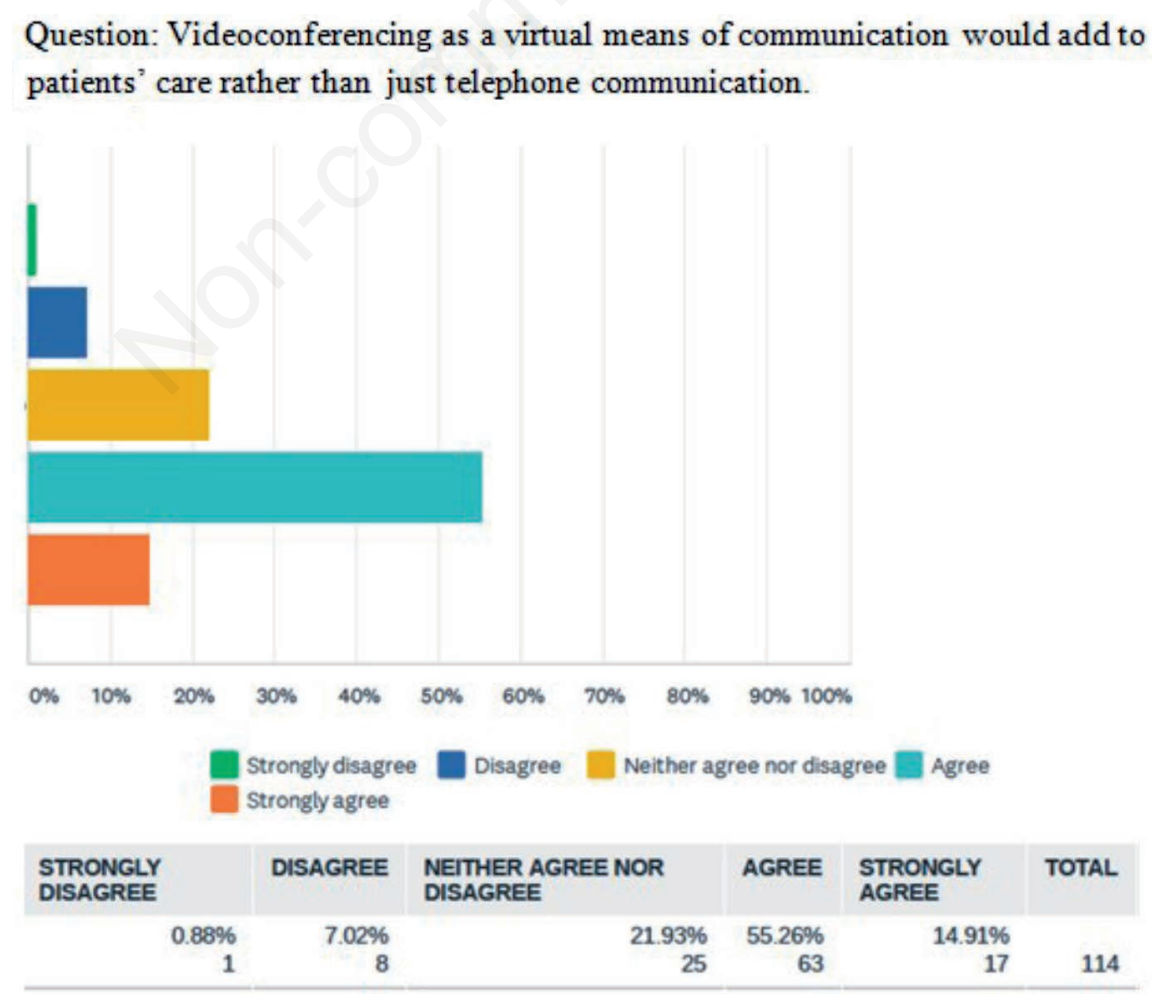

Figure 5. Videoconferencing as a means of communication. 


\section{Implications for research and practice}

This survey can act as a starting point for further research that delves deeper into the varying forms of telemedicine consultation used across different hospitals in the UK.

The technical issues addressed in this survey suggest an urgent adoption of mandatory training for all HCPs expected to use telemedicine. This will be positively reflected on patients' safety and care. This training should include the latest updated GMC guidance concerning remote consultation, and local protocols should be put in place.

NHS trusts and hospitals need to invest more in virtual clinics and telemedicine. The investments should be both in infrastructure (well equipment clinics, reliable internet connections for example) and investing in cyber security and IT support desks. Proper clinical coding of remote consultation services and a fair NHS tariff for these services will determine the size of the financial investments in telemedicine and e-health.

Having clear feedback from the patients using this service is crucial. This incorporates the patient as a partner in the consultation process and involves them in the decision making. Identifying areas of weaknesses and improving them will maintain the quality of service expected.

Further areas of interest would be to look at the impact telemedicine has on medical training. As traditionally face to face consultations are an opportunity for bedside teaching, these opportunities will potentially be reduced with less patient contact.

\section{Conclusions}

The swift change of practice to telemedicine during the COVID-19 pandemic, and the expectations of further waves of the virus, has left no doubt that telemedicine is here to stay. Health Care Professionals' reported adopting new approaches with little training but in general favourable perception. Telemedicine can be used for selected groups of patients in the post COVID-19 era, and the HCPs carrying that should have the sufficient experience and knowledge expected to operate these clinics.

\section{References}

1. WHO. Global Observatory for eHealth. Available from: https:// www.who.int/goe/publications/goe_telemedicine_2010.pdf

2. WHO. Virtual Press Conference on COVID-19. Available from: https://www.who.int/docs/default-source/coronaviruse/transcripts/who-audio-emergencies-coronavirus-press-conferencefull-and-final-11 mar2020.pdf?sfvrsn=cb432bb3_2

3. Anderson R, Heesterbeek H, Klinkenberg D, Hollingsworth T. How will country-based mitigation measures influence the course of the COVID-19 epidemic? Lancet 2020;395: 931-4.

4. MDU [Internet]. Practical advice on consulting with patients remotely. The Medical Defence Union. Available from: https://www.themdu.com/guidance-and-advice/podcasts-and-videos/practical-advice-on-consulting-withpatients-remotely

5. General Medical Council [Internet]. Remote Consultations. General Medicine Council; 2020. Available from: https:// www.gmc-uk.org/ethical-guidance/ethical-hub/remoteconsultations

6. MDU [Internet]. Lifting the Medico-legal burden during the COVID-19 pandemic. The Medical Defence Union; 2020. Available from: https://www.themdu.com/guidance-andadvice/latest-updates-and-advice/temporary-changes-to-theway-nhs-complaints-are-handled

7. Brant H, Atherton H, Ziebland S. Using alternatives to face-toface consultations: a survey of prevalence and attitudes in general practice. Br J Gen Pract 2016;66:e460-6.

8. Hjelm NM, Benefits and drawbacks of telemedicine. J Telemed Telecare 2005;11:60-70.

9. Egea J, González M, Menéndez M. eHealth usage patterns of European general practitioners: a five-year (2002-2007) comparative study. Int J Med Inform 2010;79:539-53.

10. Bertelsen P, Petersen LS. Danish citizens and general practitioners' use of ICT for their mutual communication. Stud Health Technol Inform 2015; 216:376-9.

11. Ruiz M, Maria D, Sans M, Giménez N. Implementing technology in healthcare: insights from physicians. BMC Med Inform Decis Mak 2017 27;17:92.

12. Kiberu V, Scot R, Mars M. Assessment of health provider readiness for telemedicine services in Uganda. Health Inf Manag 2019;48:33-41.

13. Health Education England. The future of primary care: creating teams for tomorrow. Report by the Primary Care Workforce Commission; 2015. Accessed on: 27 April 2016. Available from: https://ueaeprints.uea.ac.uk/id/eprint/55636/1/The_future_of_pr imary_care.pdf

14. Carter M, Fletcher E, Sansom A et al. Feasibility, acceptability and effectiveness of an online alternative to face-to-face consultation in general practice: a mixed-methods study of webGP in six Devon practices. BMJ Open 2018;8:e018688. 